\title{
Quantum transport in self-similar graphene carpets
}

\author{
G. Bouzerar $\odot^{*}$ \\ CNRS, Université Claude Bernard Lyon 1, 69622 Lyon, France \\ D. Mayou ${ }^{\circ}$ \\ Institut Néel, CNRS, 38000 Grenoble, France
}

(Received 30 March 2020; revised 26 May 2020; accepted 11 June 2020; published 13 July 2020)

\begin{abstract}
Fractals, a fascinating mathematical concept made popular in the 1980 s, has remained for decades mainly a beautiful scientific curiosity. With the tremendous advances in nanofabrication techniques, such as nanolithography, it has become possible to design self-similar materials with fine structures down to nanometer scale. Here we investigate the effects of self-similarity on quantum electronic transport in graphene Sierpinski carpets. We find that a gap opens up in the electron spectrum, in the middle of which lies a flat band of zero-energy modes. Despite the vanishing velocity of these states, a supermetallic phase is revealed at the neutrality point with a conductivity that coincides within a few percent with $\sigma_{0}=\frac{4 e^{2}}{\pi h}$. For Fermi energy located in the valence or conduction bands and in the presence of a small inelastic scattering rate, the system stays metallic and the transport appears to be strongly anisotropic.
\end{abstract}

DOI: 10.1103/PhysRevResearch.2.033063

The rapid progress in nanofabrication techniques such as nanolithography, molecular engineering, and threedimensional printing has made it possible to design complex two- and three-dimensional multiscale and self-similar materials with fine structures down to nanometer scale [1-4]. Self-similar materials have already been in use in several areas such as fractal antennas [5], photonic crystal waveguides [6], or even heat transfer devices [7]. Possibilities to design and grow at will this new class of materials open pathways for the exploration of new exotic physical phenomena that may have remarkable technological spinoffs. The rapidly growing field of cold atoms on optical or artificial lattices also offers a platform to address these fundamental issues by directly tuning the physical parameters of model Hamiltonians [8-11]. Recent theoretical studies on quantum effects in fractal lattices have been focusing on, for instance, the Hall effect [12-14], plasmon confinement [15], and topological phases [16]. In these studies the host material of the fractal structure is a simple square lattice. In this work we investigate how transport is affected by the self-similarity of the underlying lattice in one of the most remarkable two-dimensional material of the 21st century, graphene [17-20].

This one-atom-thick material holds great potential in various technological fields. It is a zero-gap semiconductor with high mobility at room temperature [21,22], it displays a huge thermal conductivity, it is extremely flexible while being

\footnotetext{
*georges.bouzerar@univ-lyon1.fr
}

Published by the American Physical Society under the terms of the Creative Commons Attribution 4.0 International license. Further distribution of this work must maintain attribution to the author(s) and the published article's title, journal citation, and DOI. stronger than steel, and it is impermeable to gas and liquids. Among its plethora of astonishing physical properties, quantum electronic transport is certainly one of the most intriguing and intensely debated [23-34]. Chirality, which results from the bipartite nature of the graphene lattice, plays a key role in its unconventional electronic transport properties, such as Klein tunneling $[35,36]$ or the existence of a conductivity minimum at the neutrality point. The effects of disorder such as $\mathrm{C}$ vacancies, short- and long-range on-site potentials, adsorbates or resonant impurities on the one-particle spectrum, and transport of the massless Dirac fermions have been the main focus of several numerical studies [27,30-32]. However, no clear consensus on the nature of the transport properties at the neutrality point could emerge. Recently, based on large-scale numerical calculations, it has been shown unambiguously that in the presence of vacancies (up to $1 \%$ ) $\sigma(0)$ remains identical to that of pristine graphene, e.g., $\sigma_{0}=\frac{4 e^{2}}{\pi h}$ [34].

In this study the issue of quantum transport in self-similar graphene Sierpinski carpets (GSCs) is addressed. We investigate the interplay between the chirality of the massless Dirac fermions, leading to the unconventional quantum transport in the host compound and the fractal nature of the spectrum resulting from self-similarity. Here we ignore the effects of disorder such as dislocation, rough edges, vacancies, or extended defects that may have an impact on transport [37-41]

Electrons on GSCs are modeled by a nearest-neighbor tight-binding Hamiltonian that reads

$$
\hat{H}=-t \sum_{\langle i j\rangle, s} c_{i s}^{\dagger} c_{j s}+\text { H.c. }
$$

where $t=2.7 \mathrm{eV}$ is the hopping integral, $\langle i j\rangle$ denotes nearestneighbor pairs of $\mathrm{C}$ atoms, and $c_{i s}^{\dagger}$ creates an electron with spin $s$ in the $\pi$ orbital at site $\mathbf{R}_{i}$. Here we ignore the 


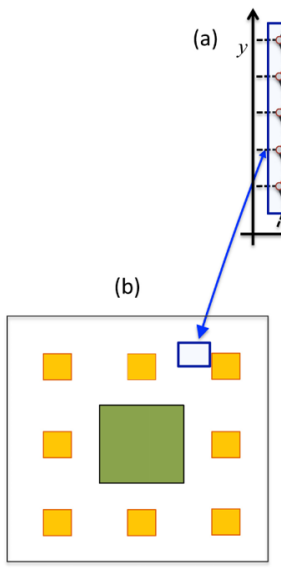

$L \times L$

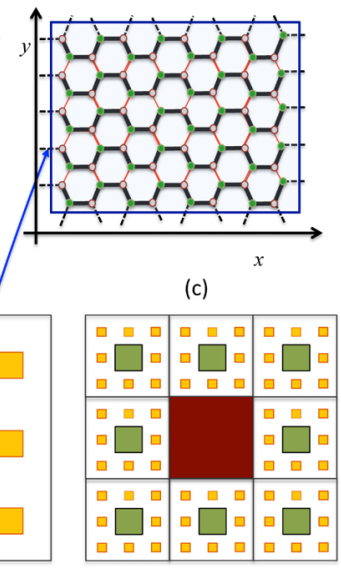

3LX $3 \mathbf{L}$

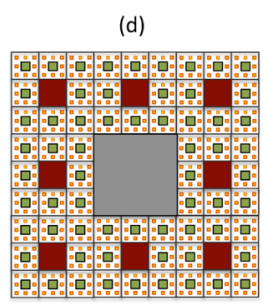

$3^{2} L \times 3^{2} L$
FIG. 1. Illustration of the GSC, from fractal level 2 to 4 . The removed squares produce armchair edges in the $x$ direction and zigzag along the $y$ direction.

next-nearest-neighbor hopping of the order of $10 \%$ that breaks particle-hole symmetry. The GSCs as illustrated in Fig. 1 are obtained from a square piece of graphene of size $3^{i_{c}+1} a(a$ is the nearest-neighbor C-C distance) on which Sierpinski masks are applied iteratively. In what follows we make use of the notation $i_{c}$ and $f$, where $L=L_{x}=L_{y}=3^{i_{c}+1} a$ is the system size and $f$ stands for the degree of fractalization, which varies from 0 (pristine) to its maximum value $f_{\max }=i_{c}$. For $f=0$, the system embodies $N_{S}=\frac{4}{3 \sqrt{3} a^{2}} L_{x} L_{y}$ of $\mathrm{C}$ atoms. Periodic boundary conditions in both the $x$ and $y$ directions (see Fig. 1) are used in the whole study. The smallest system considered corresponds to $i_{c}=3$ and the largest to $i_{c}=7$; they contain about 5000 and $33 \times 10^{6} \mathrm{C}$ atoms, respectively. Here we focus our attention on the GSC series corresponding to $i_{c}-f=0$, the maximally fractalized Sierpinski carpets. As a future prospect, one could also study the transport in other self-similar series such as $i_{c}-f=1,2, \ldots$ [42]. The conductivity along $x$ direction is given by the Kubo formula

$$
\sigma(E)=\frac{e^{2} \hbar}{\pi \Omega} \operatorname{Tr}\left[\operatorname{Im} \hat{G}_{\eta}(E) \hat{v}_{x} \operatorname{Im} \hat{G}_{\eta}(E) \hat{v}_{x}\right] .
$$

The current operator defined by $\hat{v}_{x}=-\frac{i}{\hbar}[\hat{x}, \hat{H}]$ is

$$
\hat{v}_{x}=-i \frac{a t}{\hbar} \sum_{i \in A, l, s} \alpha_{l} c_{\mathbf{R}_{i} s}^{\dagger} c_{\mathbf{R}_{i}+\delta_{l} s}+\text { H.c. }
$$

The sum runs over atoms of sublattice $A$ only; $\boldsymbol{\delta}_{l}$ are the vectors connecting a given atom to its three nearest neighbors on sublattice $B$. In the current operator expression, $\alpha_{l}=1,-\frac{1}{2}$, and $-\frac{1}{2}$ for $l=1,2$, and 3 , respectively. The Green's function $\hat{G}_{\eta}(E)=(E+i \eta-\hat{H})^{-1}, \Omega$ is the Sierpinski carpet area, and $\eta$ mimics an energy-independent inelastic scattering rate with a characteristic timescale $\tau_{\text {in }}=\frac{\hbar}{\eta}$. The calculations are done using the Chebyshev polynomial Green's function (CPGF) method $[34,43]$ that allows (i) large-scale calculations as it requires a modest amount of memory and (ii) a CPU cost that increases linearly with the system size $N_{S}$. This is in contrast to the exact diagonalization (ED) method that needs a memory scaling as $N_{S}^{2}$ and CPU time as $N_{S}^{3}$. The CPGF method has

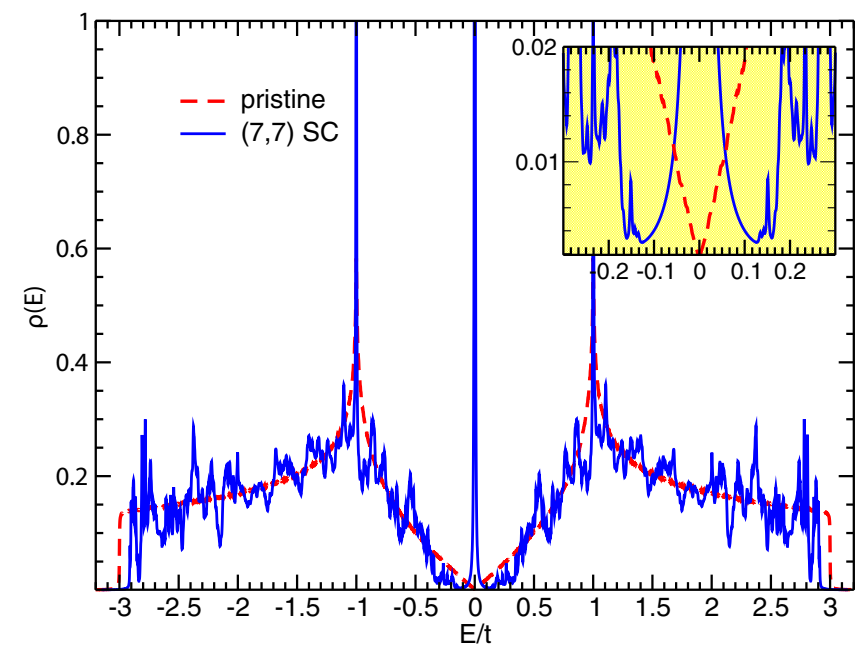

FIG. 2. Density of states calculated for the $(7,7)$ graphene Sierpinski carpet compared to that of pristine graphene (red dashed).

proven to be a powerful tool to address the nature of the magnetic couplings in disordered materials [44,45]. Notice that, in the same spirit as the CPGF method, the conductivity could be calculated by quantum wave packet dynamics as well $[31,32,46]$. Figure 2 depicts the electronic density of states (DOS) $\rho(E)=-\frac{1}{\pi N_{S}} \operatorname{Tr}\left[\operatorname{Im} \hat{G}_{\eta}(E)\right]$ as a function of energy for the $(7,7)$ GSC. Notice that we have checked, for the stochastic evaluation of the trace, that convergence was reached. More precisely, we have used a few dozen random vectors and a large number of Chebyshev polynomials, 20000 . First, we observe complex fluctuating substructures that result from the fractal nature of the eigenspectrum. Sharp peaks are visible at $E= \pm t$, corresponding to the Van Hove singularities in pristine graphene and a third one at $E=0$ that results from the removal of $\mathrm{C}$ atoms. This peak corresponds to a flat band of zero-energy modes (ZEMs). We have indeed checked by ED calculations on smaller systems that the eigenvalues are, within numerical accuracy, exactly zero. In the CPGF calculations, from the $E=0$ peak weight in the DOS we have extracted a number of ZEM $N_{\text {ZEM}}$. It is found to coincide with $\left|N_{A}-N_{B}\right|$, with $N_{A}\left(N_{B}\right)$ the number of atoms on sublattice $A(B)$, as expected for bipartite lattices [28,47]. The extracted density of ZEM modes ( $\left.x_{\text {ZEM }}\right)$ in the GSC is approximately 0.052 . Interestingly, we find a gap $\Delta$ between the conduction (valence) band and the flat band as can be seen more clearly in the inset. Its value is approximately $0.135 t$. Both $(6,6)$ and $(5,5)$ GSCs give the same value. Thus, in GSCs, the ZEM band is an impurity band. This contrasts with the gapless spectrum of randomly distributed vacancies in graphene, unless vacancies are created on the same sublattice [28]. Here Sierpinski masks produce an imbalance between $N_{A}$ and $N_{B}$, but 2-coordinated $\mathrm{C}$ atoms exist on both sublattices. Thus, the gap results only from the self-similar structure of the GSC.

It is important to emphasize that in the single-hole case $(f=1)$ for which the density of removed atoms is $1 / 9$, the spectrum is gapless in the thermodynamic limit. As $i_{c}$ increases, both the gap and the ZEM peak weight decay as $1 / L$. Hence, we recover the physics of the pristine compound. This would be the same for $f=2$ and so on. To address 


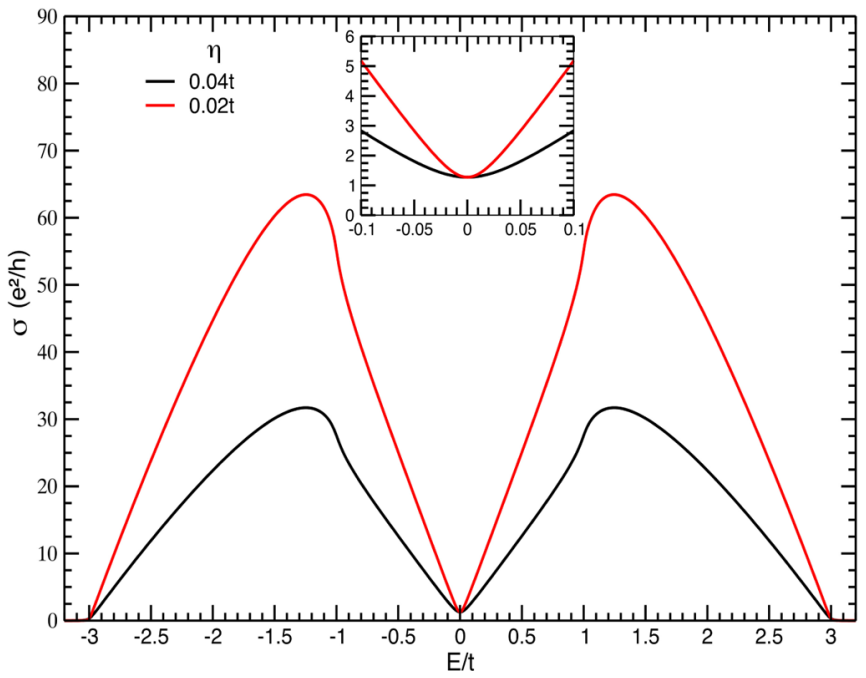

FIG. 3. Conductivity $\sigma(E)$ as a function of $E$ in the pristine case (analytical calculations) for two different values of the inelastic scattering rate $\eta=0.02 t$ and $0.04 t$. The inset shows a close-up in the vicinity of $E=0$; as expected, $\sigma(0)$ is $\frac{4}{\pi} e^{2} / h$ within less than $1 \%$. Away from the Dirac point $\sigma(E) \propto 1 / \eta$.

properly the effects of self-similarity, the calculations that allow a direct comparison between different GSCs should be performed at fixed $i_{c}-f$.

Before we proceed further with the electronic conductivity in self-similar Sierpinski carpets, we briefly recall the results for pristine graphene. In this case, the analytical calculation is relatively straightforward. The conductivity for two different values of $\eta$ is depicted in Fig. 3. Away from the Dirac point, $\sigma(E)$ scales as $1 / \eta$, as expected in standard materials when intraband transitions dominate. At $E=0$ precisely, the conductivity is independent of $\eta$ and coincides with $\frac{4}{\pi} \frac{e^{2}}{h}$. Notice that at this point, intraband and interband transitions contribute equally to $\sigma(0)$.

Let us now examine how Sierpinski carpet masks alter the transport properties. For that purpose, we calculate the DC conductivity $\sigma(E)$ in both the $x$ and $y$ directions. The results for a fixed $\eta$ are depicted in Fig. 4. The number of random vectors $N_{R}$ used for the trace calculation is 500, 100, and 10 as the system size increases. The number of Chebyshev polynomials kept is $M=2000$, leading to an $M \times M$ matrix for the moments. It has been checked that both $N_{R}$ and $M$ were sufficient to reach convergence. First, we observe a unexpected anisotropic conductivity in the GSC. Besides restricted regions where they almost coincide, $\sigma_{y y}$ is smaller than $\sigma_{x x} ; \sigma_{x x}$ is even found one order of magnitude larger than $\sigma_{y y}$ for Fermi energy in the vicinity of $\pm 0.2 t$. At first glance, it is astonishing that $\sigma_{x x} \geqslant \sigma_{y y}$ since the edges of the removed square are zigzag in the $y$ direction and armchair in the $x$ direction. As it is well known, the nature of the edge in graphene nanoribbons (GNRs) has a drastic impact on transport and magnetism [48-50]. Zigzag edges induce flatband magnetic moment formation and favor a metallic behavior, while armchair GNRs are often semiconductors or insulators. Moreover, very-small-size effects are found and the calculated conductivity for $(6,6)$ and $(7,7)$ GSCs almost

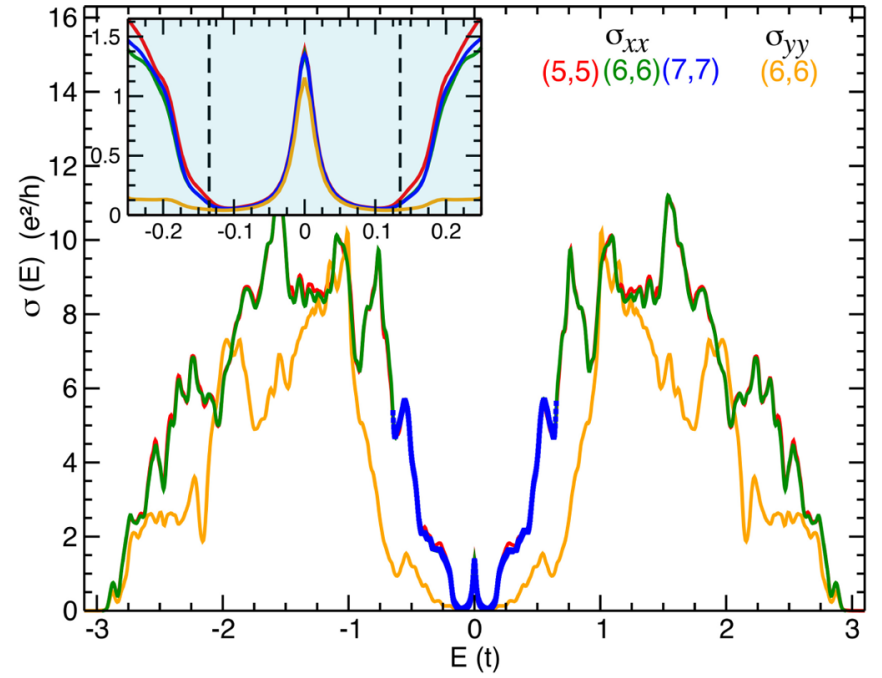

FIG. 4. Conductivity ( $x$ and $y$ directions) at $T=0 \mathrm{~K}$ as a function of the energy. Calculations for the $(7,7)$ GSC are done in a restricted region around $E=0$. The inelastic scattering rate is $\eta=$ $0.01625 t$. Along the $x$ axis, $\sigma(0)=1.39,1.37$, and $1.355 \frac{e^{2}}{h}$ for $(5,5)$, $(6,6)$, and $(7,7)$, respectively, and in the $y$ direction $\sigma(0)=1.165 \frac{e^{2}}{h}$ for the $(6,6) \mathrm{GSC}$. The inset magnifies the data around $E=0$.

coincide with each other. Notice that the carpet size (in units of $a$ ) varies from 729 for the $(5,5), 2187$ for the $(6,6)$, and 6561 for the $(7,7)$ GSCs. For Fermi energy in the conduction band (or valence band) and in the presence of a small $\eta$, the GSC remains metallic in the whole energy range. In addition, due to the fractal nature of the eigenspectrum, rich structures and multiple peaks are visible. In the vicinity of $E=0, \sigma(E)$ drops rapidly because of the gap and has a Lorentzian shape. Along the $x$ axis, the conductivity $\sigma(0)$ is $1.39 \frac{e^{2}}{h}, 1.37 \frac{e^{2}}{h}$, and $1.35 \frac{e^{2}}{h}$ for the $(5,5),(6,6)$, and $(7,7)$ GSCs, respectively. In the $y$ direction, $\sigma(0)=1.165 \frac{e^{2}}{h}$ for the $(6,6)$ GSC while for $(5,5)$ we have found $1.15 \frac{e^{2}}{h}$ (not shown). These results indicate a relatively slow convergence towards the universal value $\sigma_{0}$. Thus, despite the gap between the ZEM impurity flatband and the valence and conduction bands, the conductivity remains fully unaltered at $E=0$. It is worth noting that for randomly distributed vacancies on the same sublattice that induces a gap in the spectrum, $\sigma(E)$ was found to vanish at the neutrality point [31]. As discussed in Ref. [34], this insulating phase may result from difficulties to get converged results because of the singular density of sates. Thus, it would be of great interest to investigate the transport for this particular case with the CPGF method [51].

In Fig. 5 the effects of varying the inelastic scattering rate $\eta$ is illustrated for the $(5,5)$ GSC. We first focus on energies far from the neutrality point, e.g., $|E| \geqslant \Delta$. As $\eta$ decreases and because of the fractal nature of the spectrum, we observe (i) more and more fine structures and multiple peaks in $\sigma(E)$ and (ii) regions where it increases, alternating with a narrow energy interval where it remains insensitive. In the GSC, the conductivity is never Drude-like since one would expect a $1 / \eta$ variation of $\sigma(E)$ as in the pristine graphene (Fig. 3). Figure 5 also reveals that the conductivity at the neutrality point is 


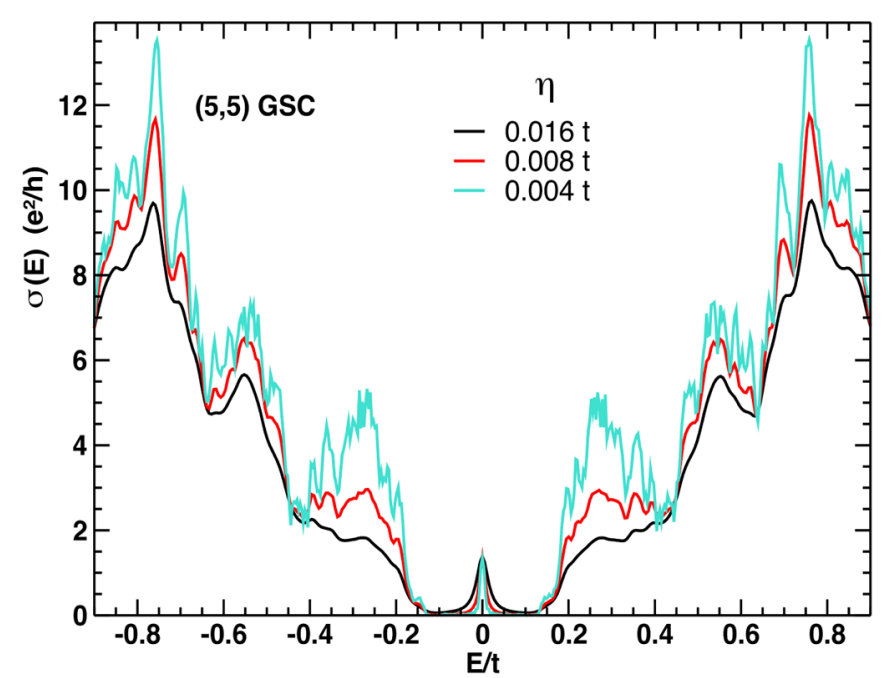

FIG. 5. Conductivity in the $x$ direction at $T=0 \mathrm{~K}$ as a function of $E$ for three different values of $\eta: \eta=0.004 t, 0.008 t$, and $0.016 t$. Here $\sigma(0)=1.37 \pm 0.02 \frac{e^{2}}{h}$; thus it is almost insensitive to $\eta$.

insensitive to $\eta: \sigma(0)=1.37 \pm 0.02 \frac{e^{2}}{h}$ for the $(5,5)$ GSC. In addition, in the gap region $(|E| \leqslant \Delta)$, the conductivity $\sigma(E)$ gets narrower and narrower as $\eta$ decreases and can be nicely fitted by a Lorentzian of width $\eta$.

With the support of exact diagonalization calculations, we propose to analyze in more detail the conductivity at the neutrality point. For $\eta \ll \Delta$, in the $x$ direction $\sigma(0)$ reduces to

$$
\sigma(0)=\frac{32}{3 \sqrt{3}} \frac{e^{2}}{h} \frac{1}{N_{S}} \sum_{\beta} C_{x, \beta},
$$

where the dimensionless $C_{x, \beta}$ is defined by

$$
C_{x, \beta}=\frac{\hbar^{2}}{a^{2}} \sum_{\alpha, \lambda= \pm} \frac{\left|\left\langle\Psi_{\beta}\left|\hat{v}_{x}\right| \Phi_{\alpha}^{\lambda}\right\rangle\right|^{2}}{E_{\alpha}^{2}},
$$

where $\left|\Psi_{\beta}\right\rangle$ are the ZEM eigenstates $\left(E_{\beta}=0\right)$ and $\left|\Phi_{\alpha}^{\lambda}\right\rangle$ those of the valence $(\lambda=-)$ and conduction $(\lambda=+)$ bands with energy $\pm E_{\alpha}\left(\left|E_{\alpha}\right|>\Delta\right)$, respectively. In the limit of vanishing $\eta(\eta \ll \Delta)$, neither interband nor intraband transitions with matrix elements $\left\langle\Phi_{\alpha}^{\lambda}\left|\hat{v}_{x}\right| \Phi_{\alpha^{\prime}}^{\lambda^{\prime}}\right\rangle$ can contribute to $\sigma(0)$ because of the gap. On the other hand, the flat intraband at $E=0$ cannot contribute either because $\left\langle\Psi_{\beta}\left|\hat{v}_{x}\right| \Psi_{\beta^{\prime}}\right\rangle$ are all zeros. Indeed, $\left|\Psi_{\beta}\right\rangle$ and $\hat{v}_{x}\left|\Psi_{\beta^{\prime}}\right\rangle$ are orthogonal to each other and they belong to the two different sublattices. Thus, $\sigma(0)$ consists only of interband transitions between the ZEM impurity band and the valence and conduction bands and a finite conductivity arises despite the presence of the gap. This feature is rather unusual. In standard systems and for $E_{F}$ in the impurity band (localized states), the conductivity is controlled by intraband transitions only and decays as $\eta$ decreases. However, in quasicrystals and approximants the scenario is different. For instance, in icosahedral quasicrystals, the diffusion coefficient is essentially controlled by interband processes, which explains the nonstandard transport properties observed [52-54].

To improve our physical understanding and get a better intuitive picture of the nature of the electronic transport in (a)

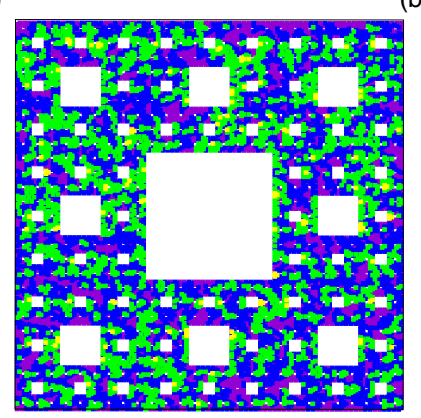

(b)

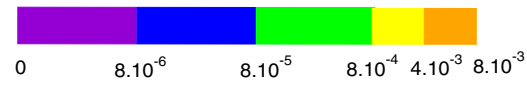

FIG. 6. Local charge density of (a) a typical ZEM state $\left|\Psi_{\beta}\right\rangle$ and (b) $\hat{v}_{x}\left|\Psi_{\beta}\right\rangle$ obtained from exact diagonalization of a $(4,4)$ GSC that contains $26833 \mathrm{C}$ atoms.

GSCs, we propose to have a close look at the local charge distribution of one typical ZEM state $\left|\Psi_{\beta}\right\rangle$ and its corresponding $\hat{v}_{x}\left|\Psi_{\beta}\right\rangle$. Because it requires the eigenstate, the calculations are performed by a direct exact diagonalization of the $(4,4)$ GSC Hamiltonian. Beyond this size the calculations would be too cumbersome. The results are illustrated in Fig. 6. This figure immediately reveals that both states are rather extended. This feature sheds light on why the overlaps between $\hat{v}_{x}\left|\Psi_{\beta}\right\rangle$ and the extended valence- or conduction-band states $\left|\Phi_{\alpha}^{\lambda}\right\rangle$ lead to a finite $\sigma(0)$ notwithstanding the existing gap. In addition, to allow a visualization of the real-space contributions to $\sigma(0)$, we define the weighted quantity

$$
\Sigma_{x}(\mathbf{r})=\sum_{\beta} C_{x, \beta}\left|\left\langle\Psi_{\beta} \mid \mathbf{r}\right\rangle\right|^{2},
$$

which is plotted in Fig. 7 for the $(4,4)$ GSC. First, as can be clearly seen, $\Sigma_{x}(\mathbf{r})$ reflects the self-similar structure of the GSC. Only atoms of sublattice $A$ are shown, since $\left|\left\langle\Psi_{\beta} \mid \mathbf{r}\right\rangle\right|^{2}$

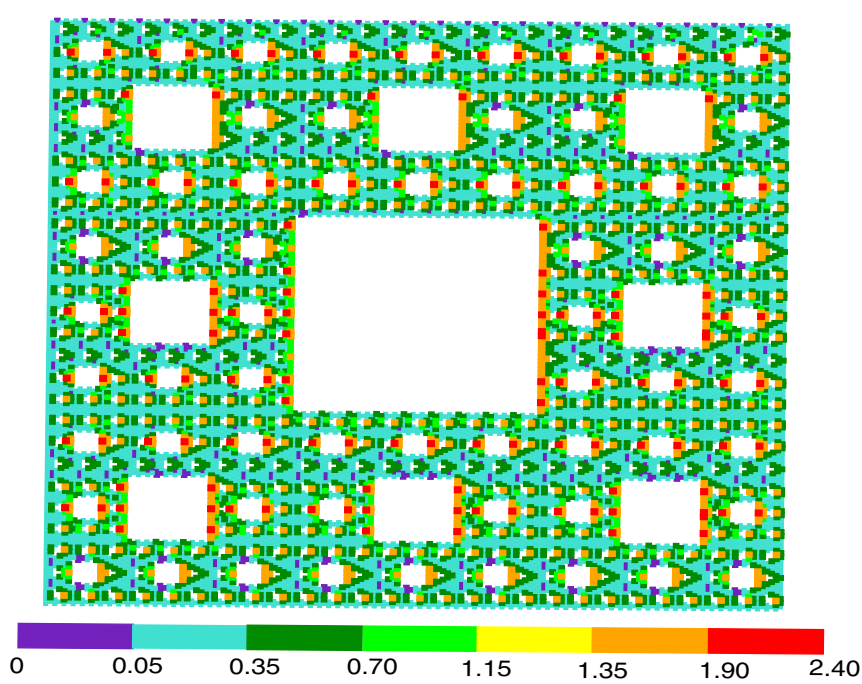

FIG. 7. Two-dimensional plot of the local contribution $\Sigma_{x}(\mathbf{r})$ to the conductivity $\sigma(0)$ as defined in the text, obtained from the exact diagonalization of the $(4,4)$ GSC Hamiltonian. 


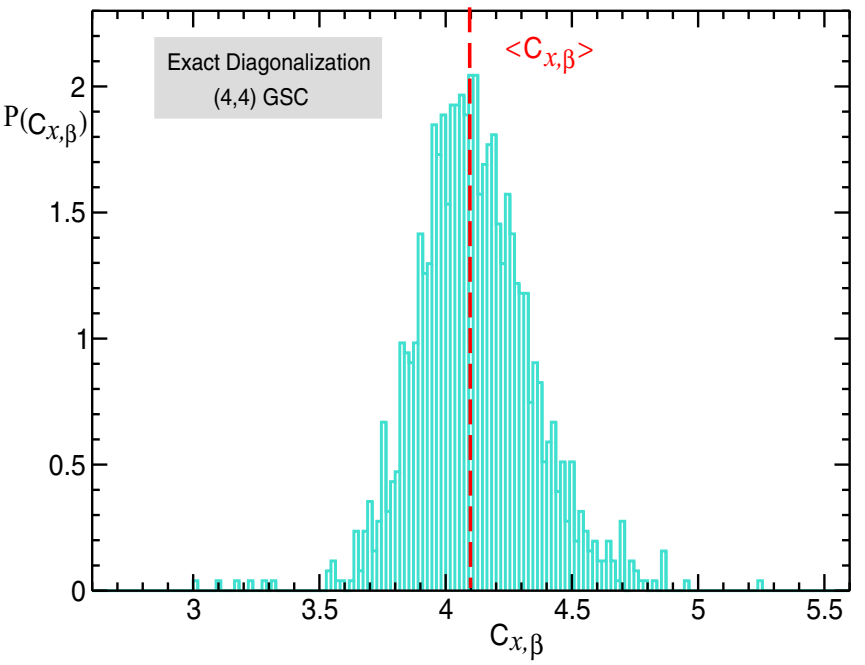

FIG. 8. Probability distribution of $C_{x, \beta}$, as defined in the text, calculated from exact diagonalization of the $(4,4)$ GSC Hamiltonian. Here $\left\langle C_{x, \beta}\right\rangle$ stands for the average value.

is exactly zero on sublattice $B$. Away from the pores, the weight is found to be relatively small. It can be seen that the dominating contributions to $\sigma(0)$ originate from the removed square edges. Notice also that the asymmetric local contribution $\Sigma_{x}(\mathbf{r})$ is much larger for $\mathrm{C}$ atoms belonging to the zigzag edges than to the armchair edges. This feature reflects the fact the the ZEM local charge density is larger along the $y$-direction edges of the removed squares, as can be seen in Fig. 6. Note that one would expect, upon switching the electron-electron interaction, the formation of magnetic moments on the sides of the removed squares parallel to the $y$ axis only.

Finally, we propose now to analyze, more quantitatively, the probability distribution of the dimensionless $C_{x, \beta}$ as obtained from the exact diagonalization of the $(4,4)$ GSC Hamiltonian. The result is depicted in Fig. 8. This plot displays a relatively narrow distribution with a mean value of $\left\langle C_{x, \beta}\right\rangle=4.1$ and width of approximately 0.5 . From Eq. (4) and recalling that the density of ZEM modes is $x_{\text {ZEM }}=$ 0.052 , we immediately get $\sigma(0)=1.315 \frac{e^{2}}{h}$, which coincides with $\sigma_{0}$ within less than $3 \%$. Notice that the diffusivity $D(E)$ can also be straightforwardly obtained from the well known Einstein formula, which reads $D(E)=\frac{\sigma(E)}{e^{2} \rho(E)}$. Here, because of the gap, in the vicinity of $E=0$, the density of states simplifies to $\rho(E)=\frac{N_{\mathrm{ZEM}}}{\pi \Omega} \frac{\eta}{E^{2}+\eta^{2}}$. From Eqs. (2) and (5) and for $|E|, \eta \ll \Delta$, the conductivity can rewritten $\sigma_{x}(E)=$ $\frac{4 e^{2} \hbar}{\pi \Omega} \sum_{\alpha, \lambda= \pm, \beta} \frac{\left|\left\langle\Psi_{\beta}\left|\hat{v}_{x}\right| \Phi_{\alpha}^{\lambda}\right\rangle\right|^{2}}{\left(E_{\alpha}-E\right)^{2}+\eta^{2}} \eta \frac{\eta}{E^{2}+\eta^{2}}$. After inserting the DOS expression, one gets the diffusivity that reads

$$
D_{x}(E)=\frac{4 a^{2}}{\hbar}\left\langle C_{x, \beta}\right\rangle \eta .
$$

Thus, the diffusivity is proportional to $\eta$ at the neutrality point. This is in contrast with the $1 / \eta$ dependence of standard metallic materials for which $D=\frac{\hbar v_{F}^{2}}{2 \eta}\left(v_{F}\right.$ being the Fermi velocity). Because the diffusivity can be written $D=\frac{L(t)^{2}}{t}$, this allows one to extract a typical length scale $L_{d}=2 \sqrt{\left\langle C_{x, \beta}\right\rangle} a \approx$ $4 a$, where $\pi L_{d}^{2}$ could be interpreted as the averaged surface occupied by a ZEM eigenstate on the GSC.

In summary, by means of the Chebyshev polynomial Green's function and exact diagonalization methods, we have investigated the effects of self-similarity on quantum electronic transport in graphene Sierpinski carpets. We have found that a finite gap opens up in the electron spectrum, in the middle of which lies a flatband of the zero-energy mode. Although ZEM states have a vanishing velocity, a supermetallic phase is found at $E_{F}=0$; the corresponding conductivity is independent of the inelastic scattering rate and coincides within few percent with the universal $\frac{4 e^{2}}{\pi h}$. Despite the gap, $\sigma(0)$ originates only from interband transitions between the ZEM impurity band and the valence or conduction bands. When $E_{F}$ lies in the valence or conduction band and in the presence of a small but finite inelastic scattering rate, the graphene Sierpinski carpets show a non-Drude metallic behavior. Finally, away from the neutrality point, the transport appears to be strongly anisotropic.

We would like to thank S. Thébaud for his relevant comments and P. Mélinon for interesting discussions.
[1] J. D. Fowlkes, R. Winkler, B. B. Lewis, M. G. Stanford, H. Plank, and P. D. Rack, ACS Nano 10, 6163 (2016).

[2] A. Vyatskikh, Nat. Commun. 9, 593 (2018).

[3] S. N. Kempkes, M. R. Slot, S. E. Freeney, S. J. M. Zevenhuizen, D. Vanmaekelbergh, I. Swart, and C. M. Smith, Nat. Phys. 15, 127 (2019).

[4] E. J. W. Berenschot, H. V. Jansen, and N. R. Tas, J. Micromech. Microeng. 23, 055024 (2013).

[5] H.-X. Xu, G.-M. W. Z. Tao, and T. J. Cui, Sci. Rep. 4, 5744 (2015).

[6] W. Wen, L. Zhou, J. Li, W. Ge, C. T. Chan, and P. Sheng, Phys. Rev. Lett. 89, 223901 (2002).

[7] K. E. Enfield, J. J. Siekas, and D. V. Pence, Microscale Thermophys. Eng. 8, 207 (2004).
[8] I. Belopolski et al., Sci. Adv. 3, e1501692 (2017).

[9] I. Bloch, J. Dalibard, and W. Zwerger, Rev. Mod. Phys. 80, 885 (2008).

[10] M. Lewenstein, A. Sanpera, V. Ahufinger, B. Damski, A. Sen(De), and U. Sen, Adv. Phys. 56, 243 (2007).

[11] N. R. Cooper, J. Dalibard, and I. B. Spielman, Rev. Mod. Phys. 91, 015005 (2019).

[12] E. van Veen, S. Yuan, M. I. Katsnelson, M. Polini, and A. Tomadin, Phys. Rev. B 93, 115428 (2016).

[13] E. van Veen, A. Tomadin, M. Polini, M. I. Katsnelson, and S. Yuan, Phys. Rev. B 96, 235438 (2017).

[14] M. Fremling, M. van Hooft, C. M. Smith, and L. Fritz, Phys. Rev. Res. 2, 013044 (2020).

[15] T. Westerhout, E. van Veen, M. I. Katsnelson, and S. Yuan, Phys. Rev. B 97, 205434 (2018). 
[16] M. Brzezinska, A. M. Cook, and T. Neupert, Phys. Rev. B 98, 205116 (2018).

[17] S. Das Sarma, S. Adam, E. H. Hwang, and E. Rossi, Rev. Mod. Phys. 83, 407 (2011).

[18] A. H. Castro Neto, F. Guinea, N. M. R. Peres, K. S. Novoselov, and A. K. Geim, Rev. Mod. Phys. 81, 109 (2009).

[19] A. K. Geim and K. S. Novoselov, Nat. Mater. 6, 183 (2007).

[20] V. Fal'ko, A. Geim, S. Das Sarma, A. MacDonald, and P. Kim, Solid State Commun. 149, 1039 (2009).

[21] S. V. Morozov, K. S. Novoselov, M. I. Katsnelson, F. Schedin, D. C. Elias, J. A. Jaszczak, and A. K. Geim, Phys. Rev. Lett. 100, 016602 (2008).

[22] Y.-W. Tan, Y. Zhang, K. Bolotin, Y. Zhao, S. Adam, E. H. Hwang, S. Das Sarma, H. L. Stormer, and P. Kim, Phys. Rev. Lett. 99, 246803 (2007).

[23] K. Nomura and A. H. MacDonald, Phys. Rev. Lett. 96, 256602 (2006).

[24] T. Ando, J. Phys. Soc. Jpn. 75, 074716 (2006).

[25] E. H. Hwang, S. Adam, and S. Das Sarma, Phys. Rev. Lett. 98, 186806 (2007).

[26] V. M. Pereira, F. Guinea, J. M. B. Lopes dos Santos, N. M. R. Peres, and A. H. Castro Neto, Phys. Rev. Lett. 96, 036801 (2006).

[27] S. Yuan, H. De Raedt, and M. I. Katsnelson, Phys. Rev. B 82, 115448 (2010).

[28] V. M. Pereira, J. M. B. Lopes dos Santos, and A. H. Castro Neto, Phys. Rev. B 77, 115109 (2008).

[29] V. Häfner, J. Schindler, N. Weik, T. Mayer, S. Balakrishnan, R. Narayanan, S. Bera, and F. Evers, Phys. Rev. Lett. 113, 186802 (2014).

[30] Z. Fan, A. Uppstu, and A. Harju, Phys. Rev. B 89, 245422 (2014).

[31] A. Cresti, F. Ortmann, T. Louvet, D. Van Tuan, and S. Roche, Phys. Rev. Lett. 110, 196601 (2013).

[32] G. Trambly de Laissardiere and D. Mayou, Phys. Rev. Lett. 111, 146601 (2013).

[33] S. Gattenlöhner, W.-R. Hannes, P. M. Ostrovsky, I. V. Gornyi, A. D. Mirlin, and M. Titov, Phys. Rev. Lett. 112, 026802 (2014).

[34] A. Ferreira and E. R. Mucciolo, Phys. Rev. Lett. 115, 106601 (2015).
[35] M. I. Katsnelson, K. S. Novoselov, and A. K. Geim, Nat. Phys. 2, 620 (2006).

[36] A. F. Young and P. Kim, Nat. Phys. 5, 222 (2009).

[37] D. A. Areshkin, D. Gunlycke, and C. T. White, Nano Lett. 7, 204 (2007).

[38] T. G. Pedersen, C. Flindt, J. Pedersen, N. A. Mortensen, A.-P. Jauho, and K. Pedersen, Phys. Rev. Lett. 100, 136804 (2008).

[39] E. R. Mucciolo, A. H. Castro Neto, and C. H. Lewenkopf, Phys. Rev. B 79, 075407 (2009).

[40] E. R. Mucciolo and C. H. Lewenkopf, J. Phys.: Condens. Matter 22, 273201 (2010).

[41] J. Dauber, B. Terrés, C. Volk, S. Trellenkamp, and C. Stampfer, Appl. Phys. Lett. 104, 083105 (2014).

[42] G. Bouzerar et al. (unpublished).

[43] A. Weisse, G. Wellein, A. Alvermann, and H. Fehske, Rev. Mod. Phys. 78, 275 (2006).

[44] R. Bouzerar, D. May, U. Löw, D. Machon, P. Melinon, S. Zhou, and G. Bouzerar, Phys. Rev. B 94, 094437 (2016).

[45] H. Lee, E. R. Mucciolo, G. Bouzerar, and S. Kettemann, Phys. Rev. B 86, 205427 (2012).

[46] F. Triozon, J. Vidal, R. Mosseri, and D. Mayou, Phys. Rev. B 65, 220202(R) (2002).

[47] E. H. Lieb, Phys. Rev. Lett. 62, 1201 (1989).

[48] K. Nakada, M. Fujita, G. Dresselhaus, and M. S. Dresselhaus, Phys. Rev. B 54, 17954 (1996).

[49] K. Wakabayashi, M. Fujita, H. Ajiki, and M. Sigrist, Phys. Rev. B 59, 8271 (1999).

[50] K. Wakabayashi, Y. Takane, M. Yamamoto, and M. Sigrist, New J. Phys. 11, 095016 (2009).

[51] G. Bouzerar et al. (unpublished). Based on the present study and on the results of Ref. [34] for randomly distributed vacancies (the compensated case), we would expect for the fully uncompensated case the dc conductivity in the vicinity of $E=0$ to behave as in the GSC.

[52] G. Trambly de Laissardiere J.-P. Julien, and D. Mayou, Phys. Rev. Lett. 97, 026601 (2006).

[53] G. Trambly de Laissardiere and D. Mayou, C. R. Phys. 15, 70 (2014).

[54] G. Trambly de Laissardiere, C. Oguey, and D. Mayou, Philos. Mag. 91, 2778 (2011). 